Galois group for some class field

Abdelmalek Azizi and Mohammed Talbi 


\title{
GALOIS GROUP FOR SOME CLASS FIELD
}

\author{
ABDELMALEK AZIZI AND MOHAMMED TALBI
}

Received 24 September, 2013

\begin{abstract}
For some imaginary quartic cyclic fields $\mathbb{K}$, we will study the capitulation problem of the 2-class ideals of $\mathbb{K}$ and we will determine the structure of the Galois group of the second Hilbert 2-class field of $\mathbb{K}$ over $\mathbb{K}$.
\end{abstract}

2010 Mathematics Subject Classification: 11R27; 11R37

Keywords: cyclic quartic fields, class group, Hilbert class field

\section{INTRODUCTION}

Let $\mathbb{F} / \mathbb{L}$ a finite extension of number fields and $O_{\mathbb{L}}$ (resp. $O_{\mathbb{F}}$ ) the ring of integers of $\mathbb{L}$ (resp. of $\mathbb{F})$. We say, since Hilbert, that an ideal of $O_{\mathbb{L}}$ capitulates in $\mathbb{F}$, if it becomes principal by extension of scalars to $O_{\mathbb{F}}$, and, of course, when an ideal $\mathfrak{a}$ of $O_{\mathbb{L}}$ capitulates in $\mathbb{F}$, then the class [a] of $\mathfrak{a}$ capitulates in $\mathbb{F}$ (ie. [ $\left.\mathfrak{a} O_{\mathbb{F}}\right]$ is trivial). Therefore, the study of the capitulation problem is precisely to describe the group of all classes of ideals of $\mathbb{L}$ which capitulate in $\mathbb{F}$, where $\mathbb{F}$ is an unramified abelian extension of $\mathbb{L}$.

Proposition 1 ([8]). Let $\mathbb{G}$ a 2-group of finite order $2^{m}$ and $\mathbb{G}^{\prime}$ its derived subgroup. Then $\mathbb{G} / \mathbb{G}^{\prime}$ is of type $(2,2)$ if and only if $\mathbb{G}$ is isomorphic to one of 2-groups:

$$
\begin{aligned}
& Q_{m}=\langle\sigma, \tau\rangle \text { where } \sigma^{2^{m-2}}=\tau^{2}=a, a^{2}=1, \tau^{-1} \sigma \tau=\sigma^{-1} ; \\
& D_{m}=\langle\sigma, \tau\rangle \text { where } \sigma^{2^{m-1}}=\tau^{2}=1, \tau^{-1} \sigma \tau=\sigma^{-1} \text {; } \\
& S_{m}=\langle\sigma, \tau\rangle \text { where } \sigma^{2^{m-1}}=\tau^{2}=1, \tau^{-1} \sigma \tau=\sigma^{2^{m-2}-1} \text {; } \\
& (2,2)=\langle\sigma, \tau\rangle \text { where } \sigma^{2}=\tau^{2}=1, \tau^{-1} \sigma \tau=\sigma .
\end{aligned}
$$

Where $Q_{m}$ is the quaternionic group, $D_{m}$ the dihedral group, $S_{m}$ the semi-dihedral group of order $2^{m}$ and (2.2) is an abelian group isomorphic to $\mathbb{Z} / 2 \mathbb{Z} \times \mathbb{Z} / 2 \mathbb{Z}$.

Suppose that $\mathbb{G}$ is a 2-group of finite order $2^{m}$ such that $\mathbb{G} / \mathbb{G}^{\prime}$ is of type $(2,2)$, then $\mathbb{G}$ is isomorphic to $Q_{m}, D_{m}, S_{m}$ or $(2,2)$ defined in the Proposition 1. Let $\{\sigma, \tau\}$ generates $\mathbb{G}$ such that the relationships cited in the Proposition 1 are verified, by a simple calculation we can see that the derived subgroup $\mathbb{G}^{\prime}=[\mathbb{G}, \mathbb{G}]=\left\langle\sigma^{2}\right\rangle$ 
and $\mathbb{G}$ has three subgroups of index 2: $H_{1}=\langle\sigma\rangle, H_{2}=\left\langle\sigma^{2}, \tau\right\rangle$ and $H_{3}=\left\langle\sigma^{2}, \sigma \tau\right\rangle$. Moreover if $\mathbb{G}$ is of type $(2,2)$, then the subgroups $H_{i}$ are cyclic of order 2 , if $\mathbb{G} \simeq$ $Q_{3}$, then the subgroups $H_{i}$ are cyclic of order 4 and if $\mathbb{G}$ is isomorphic to $Q_{m}(m>$ 3), $D_{m}$ or $S_{m}$, then $H_{1}$ is cyclic and $H_{2} / H_{2}^{\prime}$ and $H_{3} / H_{3}^{\prime}$ are of type $(2,2)$ where $H_{2}^{\prime}$ (resp. $H_{3}^{\prime}$ ) is the derived subgroup of $H_{2}$ (resp. $H_{3}$ ).

Throughout the remainder of this section we denote by $M$ a number field, $M_{2}^{(1)}$ the first 2-Hilbert class field of $\mathrm{M}, \mathrm{M}_{2}^{(2)}$ the second 2-Hilbert class field of $\mathrm{M}, C_{\mathrm{M}}$ the class group of $\mathrm{M}, C_{2, \mathrm{M}}$ the 2-part of $C_{\mathrm{M}}, \mathbb{G}$ the Galois group of $\mathrm{M}_{2}^{(2)} / \mathrm{M}$ and $\mathbb{G}^{\prime}$ its derived sub-group. Then $\mathbb{G}^{\prime} \simeq \operatorname{Gal}\left(\mathrm{M}_{2}^{(2)} / \mathrm{M}_{2}^{(1)}\right)$ and $\mathbb{G} / \mathbb{G}^{\prime} \simeq \operatorname{Gal}\left(\mathrm{M}_{2}^{(1)} / \mathbf{M}\right)$, and we know from the class field theory that $\operatorname{Gal}\left(\mathrm{M}_{2}^{(1)} / \mathrm{M}\right) \simeq C_{2, \mathbb{M}}$, so $\mathbb{G} / \mathbb{G}^{\prime} \simeq C_{2, \mathbb{M}}$.

Definition 1. Let $\mathbb{F}$ be a cyclic unramified extension of $\mathbb{M}$ and $j$ is the mapping of $C_{\mathrm{M}}$ into $C_{\mathbb{F}}$ that maps to the class of an ideal $\mathfrak{a}$ of $\mathrm{M}$ the class of the ideal generated by $\mathfrak{a}$ in $\mathbb{F}$. Then the extension $\mathbb{F} / \mathbb{M}$ is called:

- of type $(A)$ if and only if \#ker $j \cap N_{\mathbb{F} / \mathrm{M}}\left(C_{\mathbb{F}}\right)>1$;

- of type $(B)$ if and only if \#ker $j \cap N_{\mathbb{F} / \mathbb{M}}\left(C_{\mathbb{F}}\right)=1$.

Now suppose that $C_{2, \mathbb{M}} \simeq \mathbb{Z} / 2 \mathbb{Z} \times \mathbb{Z} / 2 \mathbb{Z}$, then $\mathbb{G} / \mathbb{G}^{\prime} \simeq \mathbb{Z} / 2 \mathbb{Z} \times \mathbb{Z} / 2 \mathbb{Z}$ and as $\mathbb{G}$ is a 2-finite group, then $\mathbb{G}^{\prime}$ is cyclic. Thus the Hilbert 2-class field towers of $M$ ends in $\mathrm{M}_{2}^{(2)}$.

In addition, we know that if $\mathbb{G}$ has order $2^{m}$ and $\mathbb{G} / \mathbb{G}^{\prime} \simeq \mathbb{Z} / 2 \mathbb{Z} \times \mathbb{Z} / 2 \mathbb{Z}$, then $\mathbb{G}$ is isomorphic to $Q_{m}, D_{m}, S_{m}$ or to $\mathbb{Z} / 2 \mathbb{Z} \times \mathbb{Z} / 2 \mathbb{Z}$. In all these cases, we have $\mathbb{G}^{\prime}=\left\langle\sigma^{2}\right\rangle$ and the three subgroups of index 2 in $\mathbb{G}$ are: $H_{1}=\langle\sigma\rangle, H_{2}=\left\langle\sigma^{2}, \tau\right\rangle$ and $H_{3}=\left\langle\sigma^{2}, \sigma \tau\right\rangle$, and if $\mathbb{G}^{\prime} \neq 1$, then $\mathbb{M}_{2}^{(1)} \neq \mathbb{M}_{2}^{(2)}$ and $\left\langle\sigma^{4}\right\rangle$ is the only subgroup of $\mathbb{G}^{\prime}$ of index 2 .

Let $\mathbb{L}$ the subfield of $\mathbf{M}_{2}^{(2)}$ left fixed by $\left\langle\sigma^{4}\right\rangle, \mathbb{F}_{i}(i=1,2,3)$ the subfield of $\mathbf{M}_{2}^{(2)}$ left fixed by $H_{i}$ and $j_{i}$ the mapping $j$ defined for $\mathbb{F}=\mathbb{F}_{i}$.

Theorem 1 ([8]). Assume that $\mathbb{G} / \mathbb{G}^{\prime} \simeq \mathbb{Z} / 2 \mathbb{Z} \times \mathbb{Z} / 2 \mathbb{Z}$, so we have

(1) If $\mathrm{M}_{2}^{(1)}=\mathrm{M}_{2}^{(2)}$, then the fields $\mathbb{F}_{i}$ are of type $(A)$, \#ker $j_{i}=4$ for $i=1,2,3$ and $\mathbb{G} \simeq \mathbb{Z} / 2 \mathbb{Z} \times \mathbb{Z} / 2 \mathbb{Z}$

(2) If $\operatorname{Gal}(\mathbb{L} / \mathbb{M}) \simeq Q_{3}$, then the fields $\mathbb{F}_{i}$ are of type $(A)$, \#ker $j_{i}=2$ for $i=$ $1,2,3$ and $\mathbb{G} \simeq Q_{3}$;

(3) If $\operatorname{Gal}(\mathbb{L} / \mathbb{M}) \simeq D_{3}$, then the fields $\mathbb{F}_{2}$ and $\mathbb{F}_{3}$ are of type $(B)$ and $\#$ ker $j_{2}=$ $\#$ ker $j_{3}=2$. Moreover, if $\mathbb{F}_{1}$ is of type $(B)$ then $\# \operatorname{ker} j_{1}=2$ and $\mathbb{G} \simeq S_{m}$. If $\mathbb{F}_{1}$ is of type $(A)$ and $\# \operatorname{ker} j_{1}=2$, then $\mathbb{G} \simeq Q_{m}$. Finally if $\mathbb{F}_{1}$ is of type $(A)$ and \#ker $j_{1}=4$, then $\mathbb{G} \simeq D_{m}$. 
Theorem 2 ([6]). Let $\mathbb{L} / \mathbb{M}$ be an unramified cyclic extension of prime degree, then the number of classes that capitulate in $\mathbb{L} / \mathrm{M}$ is

$$
[\mathbb{L}: \mathbb{M}]\left[E_{\mathbb{M}}: N_{\mathbb{L} / \mathbb{M}}\left(E_{\mathbb{L}}\right)\right],
$$

where $E_{\mathbb{M}}$ (resp. $\left.E_{\mathbb{L}}\right)$ is the group of units of $\mathbb{M}$ (resp. of $\left.\mathbb{L}\right)$.

For more details about the capitulation problem see $[8,11,12]$.

\section{UNITS OF SOME NUMBER FIELDS}

Proposition 2 ([1]). Let $\mathbb{K}_{0}$ a number field, abelian real and $\beta$ an algebraic integer in $\mathbb{K}_{0}$, totally positive, without square factors. Assume that $\mathbb{F}=\mathbb{K}_{0}(\sqrt{-\beta})$ is a quadratic extension of $\mathbb{K}_{0}$, abelian over $\mathbb{Q}$ and $i=\sqrt{-1} \notin \mathbb{F}$. Let $\left\{\varepsilon_{1}, \varepsilon_{2}, \ldots, \varepsilon_{r}\right\}$ be a fundamental system of units of $\mathbb{K}_{0}$. We choose, without limiting the generality, units $\varepsilon_{j}$ positive. Then we have:

(1) If there is a unit of $\mathbb{K}_{0}$ such that $\varepsilon=\varepsilon_{1}^{j_{1}} \varepsilon_{2}^{j_{2}} \ldots \varepsilon_{r-1}^{j_{r-1}} \varepsilon_{r}$ (close to a permutation), where the $j_{k} \in\{0,1\}$, such that $\beta \varepsilon$ is a square in $\mathbb{K}_{0}$, then $\left\{\varepsilon_{1}, \ldots, \varepsilon_{r-1}, \sqrt{-\varepsilon}\right\}$ is a fundamental system of units of $\mathbb{F}$;

(2) Otherwise $\left\{\varepsilon_{1}, \ldots, \varepsilon_{r}\right\}$ is a fundamental system of units of $\mathbb{F}$.

Corollary 1 ([2]). Let $\mathbb{L}=\mathbb{Q}(\sqrt{-n \varepsilon \sqrt{d}})$ a cyclic extension of degree 4 over $\mathbb{Q}$, where $\varepsilon$ is the fundamental unit of $\mathbb{Q}(\sqrt{d})$ with $d$ a positive integer squarefree and $n$ a positive integer, then $\{\varepsilon\}$ is a fundamental system of units of $\mathbb{L}$.

Theorem $3([2])$. Let $\mathbb{K}_{0}=\mathbb{Q}\left(\sqrt{p}, \sqrt{p^{\prime}}\right)$ where $p$ and $p^{\prime}$ are two different primes such that $p \equiv p^{\prime} \equiv 1 \bmod 4, \varepsilon_{1}$ (resp. $\left.\varepsilon_{2}, \varepsilon_{3}\right)$ the fundamental unit of $\mathbb{Q}(\sqrt{p})$ (resp. $\left.\mathbb{Q}\left(\sqrt{p^{\prime}}\right), \mathbb{Q}\left(\sqrt{p p^{\prime}}\right)\right)$ and $\mathbb{F}=\mathbb{K}_{0}\left(\sqrt{-n \varepsilon_{1} \sqrt{p}}\right)$ where $n$ is a positive integer squarefree. Then we have:

(1) If $\varepsilon_{3}$ has norm 1 , then $\left\{\varepsilon_{1}, \varepsilon_{2}, \sqrt{\varepsilon_{3}}\right\}$ is a fundamental system of units of $\mathbb{K}_{0}$ and of $\mathbb{F}$;

(2) Otherwise, $\left\{\sqrt{\varepsilon_{1} \varepsilon_{2} \varepsilon_{3}}, \varepsilon_{2}, \varepsilon_{3}\right\}$ is a fundamental system of units of $\mathbb{K}_{0}$ and of F.

Now, using the results of M. N. Gras [4], we'll define a fundamental system of units of real cyclic extension of degree 4 over $\mathbb{Q}$. Let $\mathbb{L}=\mathrm{k}\left(\sqrt{\varepsilon_{0} \sqrt{l}}\right)$ where $l$ is a prime number congruent to 1 modulo 8 and $\varepsilon_{0}$ is the fundamental unit of $\mathrm{k}=\mathbb{Q}(\sqrt{l})$, then $\mathbb{L} / \mathbb{Q}$ is a real cyclic extension of degree 4 with Galois group $H=\langle\gamma\rangle$ and quadratic subfield k. Since $\mathbb{L}$ has conductor $F_{\mathbb{L}}=l$, we have $\mathbb{L} \subset \mathbb{Q}^{(l)}$ and there exists a character $\chi^{\prime}$ of $\operatorname{Gal}\left(\mathbb{Q}^{(l)} / \mathbb{Q}\right) \simeq(\mathbb{Z} / l \mathbb{Z})^{*}$ such that $\operatorname{ker} \chi^{\prime}=\operatorname{Gal}\left(\mathbb{Q}^{(l)} / \mathbb{L}\right)$.

Let $\chi=\chi^{\prime}+\chi^{\prime-1}$, then $\chi$ is a rational character of $\mathbb{Q}^{(l)}$ and $\mathbb{L}$ is fixed by the common kernel of $\chi^{\prime}$ and $\chi^{\prime-1}$. Let $E_{\mathbb{L}}$ be the group of units of $\mathbb{L}, E_{\chi}$ the group of $\chi$-relative units of $\mathbb{L},\left|E_{\mathbb{L}}\right|$ (resp. $\left|E_{\chi}\right|$ ) the group of absolute values of $E_{\mathbb{L}}$ (resp. 
$\left.E_{\chi}\right),\left|E^{\mathbb{Q}}\right|=\left|E_{\mathbb{L}}\right| \oplus\left|E_{\chi}\right|, Q=\left[\left|E_{\mathbb{L}}\right|:\left|E^{\mathbb{L}}\right|\right]$ and $\varepsilon_{\chi}$ a generator of $E_{\chi}$. Then we have the following result:

Theorem 4 ([4]). Let $\mathbb{L}=\mathrm{k}\left(\sqrt{\varepsilon_{0} \sqrt{l}}\right)$ where $l$ is a prime number congruent to 1 modulo 8 and $\varepsilon_{0}$ the fundamental unit of $\mathrm{k}=\mathbb{Q}(\sqrt{l})$, then:

(1) $Q=2$;

(2) There exists $\xi$ in $E_{\mathbb{L}}$ such that $\xi^{2}= \pm \varepsilon_{0} \varepsilon_{\chi}^{1-\sigma}$ and $\left\{\xi, \xi^{\sigma}, \xi^{\sigma^{2}}\right\}$ is a fundamental system of units of $\mathbb{L}$.

Remark 1. Since $\xi^{2}= \pm \varepsilon_{0} \varepsilon_{\chi}^{1-\sigma}$, then:

(1) $\xi^{1+\sigma}= \pm \varepsilon_{\chi}$;

(2) $\xi^{1+\sigma^{2}}= \pm \varepsilon_{0}$;

(3) $\xi^{1+\sigma+\sigma^{2}+\sigma^{3}}=\varepsilon_{\chi}^{1+\sigma^{2}}=\varepsilon_{0}^{1+\sigma}$;

(4) $N_{\mathrm{k} / \mathbb{Q}}\left(\varepsilon_{0}\right)=N_{\mathbb{L} / \mathrm{k}}\left(\varepsilon_{\chi}\right)=N_{\mathbb{L} / \mathbb{Q}}(\xi)=-1$.

Lemma 1. With the same notation of Theorem $4,\left\{\xi, \xi^{\sigma}, \xi^{\sigma^{2}}\right\}$ is a fundamental system of units of $\mathbb{E}=\mathbb{L}(\sqrt{-n})$ where $n$ is an integer different from 1, relatively prime to $l$ and square-free.

Proof. By Proposition 2, to show that $\left\{\xi, \xi^{\sigma}, \xi^{\sigma^{2}}\right\}$ is a fundamental system of units of $\mathbb{F}$ it suffices to show that $n \mu$ is not a square in $\mathbb{L}$, for $\mu=\xi_{1}^{\prime j_{1}} \xi_{2}^{\prime j_{2}} \xi_{3}^{\prime}$ where $\left\{\xi_{1}^{\prime}, \xi_{2}^{\prime}, \xi_{3}^{\prime}\right\}=\left\{\xi, \xi^{\sigma}, \xi^{\sigma^{2}}\right\}$ and $j_{1}, j_{2} \in\{0,1\}$.

Indeed, if $\mu=\xi$, then if $n \xi=x^{2}$ in $\mathbb{L}$, so by calculating the norm in $\mathbb{L} / \mathrm{k}$, we find that $\xi^{1+\sigma^{2}}= \pm \varepsilon_{0}$ is a square in $\mathrm{k}$, which is impossible.

If $\mu=\xi^{\sigma}$, then if $n \xi^{\sigma}= \pm n \frac{\varepsilon_{\chi}}{\xi}=x^{2}$ in $\mathbb{L}$, so by using the norm in $\mathbb{L} / \mathrm{k}$, we find that $\pm \frac{\varepsilon_{0}^{1+\sigma}}{\varepsilon_{0}}= \pm \varepsilon_{0}^{-1}$ is a square in $\mathrm{k}$, which is absurd.

If $\mu=\xi^{\sigma^{2}}$, then if $n \xi^{\sigma^{2}}= \pm n \frac{\varepsilon_{0}}{\xi}=x^{2}$ in $\mathbb{L}$, so by calculating the norm in $\mathbb{L} / \mathrm{k}$, we find that $\pm \frac{\varepsilon_{0}^{2}}{\varepsilon_{0}}= \pm \varepsilon_{0}$ is a square in $\mathrm{k}$, which is not the case.

If $\mu=\xi^{1+\sigma}= \pm \varepsilon_{\chi}$, then if $\pm n \varepsilon_{\chi}=x^{2}$ in $\mathbb{L}$, so by calculating the norm in $\mathbb{L} / \mathrm{k}$, we find that $\varepsilon_{\chi}^{1+\sigma^{2}}=\varepsilon_{0}^{1+\sigma}=-1$ is a square in $\mathrm{k}$, which is absurd.

If $\mu=\xi^{1+\sigma^{2}}= \pm \varepsilon_{0}$, then $\pm n \varepsilon_{0}$ can not be a square in $\mathbb{L}$.

If $\mu=\xi^{\sigma+\sigma^{2}}$, then if $n \xi^{\sigma+\sigma^{2}}=x^{2}$ in $\mathbb{L}$, so by calculating the norm in $\mathbb{L} / \mathrm{k}$, we find that $\xi^{1+\sigma+\sigma^{2}+\sigma^{3}}=-1$ is a square in $\mathrm{k}$, which is not the case.

If $\mu=\xi^{1+\sigma+\sigma^{2}}$, then if $n \xi^{1+\sigma+\sigma^{2}}=x^{2}$ in $\mathbb{L}$, so by calculating the norm in $\mathbb{L} / \mathrm{k}$, we find that $\xi^{1+\sigma+\sigma^{2}+\sigma^{3}} \xi^{1+\sigma^{2}}= \pm \varepsilon_{0}$ is a square in $\mathrm{k}$, which is impossible, which completes the proof of the lemma. 


\section{Capitulation of the 2-IDEAl Class of $\mathbb{K}$ AND STRUCture of $\mathbb{G}_{2}$}

In the following, let $\mathbb{K}=\mathrm{k}\left(\sqrt{-p q \varepsilon_{0} \sqrt{l}}\right)$ where $\varepsilon_{0}$ is the fundamental unit of $\mathrm{k}=\mathbb{Q}(\sqrt{l})$ with $l$ is a prime number congruent to 1 modulo $8, p$ and $q$ two prime numbers such that $p \equiv-q \equiv 1 \bmod 4$ and $\left(\frac{p}{l}\right)=\left(\frac{q}{l}\right)=-1, \mathbb{K}_{2}^{(1)}$ the Hilbert 2-class field of $\mathbb{K}, \mathbb{K}_{2}^{(2)}$ the Hilbert 2-class field of $\mathbb{K}_{2}^{(1)}, \mathbb{G}_{2}$ the Galois group of $\mathbb{K}_{2}^{(2)} / \mathbb{K}$ and for an ideal $\mathfrak{a}$ of $\mathbb{K}$, we note [a] the class of $\mathfrak{a}$. Then, by [3], $C_{2, \mathbb{K}}$, the 2-class group of $\mathbb{K}$, is of type $(2,2)$. Using the Results of [7] for the calculation of the genus fields of an extension of degree $2^{s}$ on $\mathbb{Q}$, we find that $\mathbb{K}_{2}^{(1)}=\mathbb{K}^{(*)}=\mathbb{K}(\sqrt{p}, \sqrt{-q})$, where $\mathbb{K}^{(*)}$ is the genus field of $\mathbb{K}$, whose quadratic subfields over $\mathbb{K}$ are $\mathbb{F}_{1}=\mathbb{K}(\sqrt{p})$, $\mathbb{F}_{2}=\mathbb{K}(\sqrt{-q})$ and $\mathbb{F}_{3}=\mathbb{K}(\sqrt{-p q})$.

We study the capitulation problem of the 2-ideal classes of $\mathbb{K}$ in different sub-quadratic extension $\mathbb{F}_{i} / \mathbb{K}$ of $\mathbb{K}_{2}^{(1)} / \mathbb{K}$, and hence we determine the structure of $\mathbb{G}_{2}$.

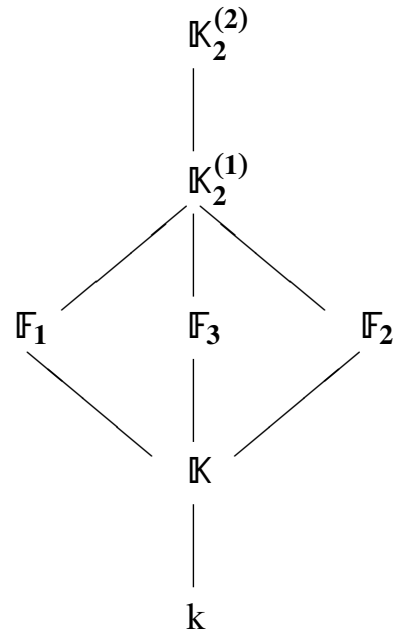

Proposition 3. Let $\mathbb{K}=\mathrm{k}\left(\sqrt{-p q \varepsilon_{0} \sqrt{l}}\right)$, $\mathcal{P}$ the prime ideal of $\mathbb{K}$ above $p$ and $Q$ that above $q$. Then the classes $[\mathcal{P}],[\mathcal{Q}]$ and $[\mathcal{P Q}]$ are of order 2 in $\mathbb{K}, C_{2, \mathbb{K}}$ is generated by the classes $[\mathcal{P}]$ and $[\mathcal{Q}]$. Also $\mathcal{P}$ capitulates in $\mathbb{F}_{1}, \mathcal{Q}$ capitulates in $\mathbb{F}_{2}$ and $\mathcal{P} Q$ capitulates in $\mathbb{F}_{3}$.

Proof. The class $[\mathcal{P}]$ has order 2 , indeed, since $p$ is inert in $\mathrm{k} / \mathbb{Q}$ and $p$ ramifies in $\mathbb{K} / \mathbb{Q}$, then $\mathcal{P}^{2}=(p)$. Assume that $\mathcal{P}=(\alpha)$ for some $\alpha$ in $\mathbb{K}$, which is equivalent to $\left(\alpha^{2}\right)=(p)$ in $\mathbb{K}$. So there is therefore a unit $\varepsilon$ of $K$ such that $p \varepsilon=\alpha^{2}$, but there exist $a$ and $b$ in k such that $\alpha=a+b \sqrt{-p q \varepsilon_{0} \sqrt{l}}$, thus $p \varepsilon=a^{2}-p q \varepsilon_{0} \sqrt{l} b^{2}+$ $2 a b \sqrt{-p q \varepsilon_{0} \sqrt{l}}$ and as $\left\{\varepsilon_{0}\right\}$ is a fundamental system of units of $\mathbb{K}$ (Corollary 1$)$ and 
$i=\sqrt{-1} \notin \mathbb{K}$, then $p \varepsilon \in \mathrm{k}$, therefore $a$ or $b=0$. If $b=0$, then $p \varepsilon=a^{2}$, thus, if $\varepsilon$ has norm 1 (the norm in $\mathrm{k} / \mathbb{Q}$ ), $p$ will be norm in $\mathrm{k} / \mathbb{Q}$ which is not the case because $\left(\frac{p}{l}\right)=-1$, if $\varepsilon$ has norm -1 we find that -1 is a square in $\mathbb{Q}$ which is impossible. Similarly, if $a=0$ we find that $\pm l$ is a square in $\mathbb{Q}$, thus $[\mathcal{P}]$ has order 2 and similarly one shows that $[Q \mathcal{Q}]$ and $[\mathcal{P} Q]$ orders are 2 , therefore $C_{2, \mathbb{K}}$ is generated by $[\mathcal{P}]$ and $[Q \mathcal{Q}]$. To show that $\mathcal{P}$ capitulates in $\mathbb{F}_{1}$, it suffices to see that $\sqrt{p} \in \mathbb{F}_{1}$ and $\left(\sqrt{p}^{2}\right)=(p)$ in $\mathbb{F}_{1}$, so $\mathcal{P}$ capitulated in $\mathbb{F}_{1}$ and even $\mathcal{Q}$ capitulated in $\mathbb{F}_{2}$ and $\mathcal{P Q}$ capitulated in $\mathbb{F}_{3}$.

Proposition 4 ([5]). Let $\mathrm{M}$ a number field which contains the m-th roots of unity, $\mathbb{L}$ a finite extension of $\mathbb{M}, \alpha \in \mathbb{M}^{*}$ and $\beta \in \mathbb{L}^{*}$. We denote by $P$ a prime ideal of $\mathbb{M}$, and $\mathcal{P}$ a prime ideal of $\mathbb{L}$ above $P$. Then

$$
\prod_{\mathcal{P}}\left(\frac{\beta, \alpha}{\mathcal{P}}\right)_{m}=\left(\frac{N_{\mathbb{L} / \mathbb{M}}(\beta), \alpha}{P}\right)_{m},
$$

where the product is taken for all prime ideals of $\mathbb{L}$ that are above $P$.

Theorem 5. Let $\mathbb{K}=\mathrm{k}\left(\sqrt{-p q \varepsilon_{0} \sqrt{l}}\right)$ where $\varepsilon_{0}$ is the fundamental unit of $\mathrm{k}=$ $\mathbb{Q}(\sqrt{l})$ with $l$ is a prime number congruent to 1 modulo $8, p$ and $q$ be two prime numbers such that $p \equiv-q \equiv 1 \bmod 4$ and $\left(\frac{p}{l}\right)=\left(\frac{q}{l}\right)=-1, \mathbb{F}_{1}=\mathbb{K}(\sqrt{p}), \mathbb{F}_{2}=$ $\mathbb{K}(\sqrt{-q})$ and $\mathbb{F}_{3}=\mathbb{K}(\sqrt{-p q})$. Then in each extension $\mathbb{F}_{i}, i \in\{1,2,3\}$, there exist exactly two classes of $C_{2, \mathbb{K}}$ which capitulate and the group $\mathbb{G}_{2}$ is quaternionic of order $2^{m}$ with $m>3$.

Proof. Let $\varepsilon_{2}$ (resp. $\left.\varepsilon_{3}\right)$ the fundamental unit of $\mathbb{Q}(\sqrt{p})$ (resp. $\left.\mathbb{Q}(\sqrt{l p})\right), \mathcal{P}$ the prime ideal of $\mathbb{K}$ above $p, \mathcal{Q}$ that above $q$, then, by Theorem $3,\left\{\sqrt{\varepsilon_{0} \varepsilon_{2} \varepsilon_{3}}, \varepsilon_{2}, \varepsilon_{3}\right\}$ is a fundamental system of units of $\mathbb{F}_{1}$, since $N_{\mathbb{F}_{1} / \mathbb{K}}\left(\sqrt{\varepsilon_{0} \varepsilon_{2} \varepsilon_{3}}\right)= \pm \varepsilon_{0}$ and $N_{\mathbb{F}_{1} / \mathbb{K}}\left(\varepsilon_{2}\right)=$ $N_{\mathbb{F}_{1} / \mathbb{K}}\left(\varepsilon_{3}\right)=-1$, then $N_{\mathbb{F}_{1} / \mathbb{K}}\left(E_{\mathbb{F}_{1}}\right)=E_{\mathbb{K}}$, therefore, by Theorem 2, we found that only two classes of $C_{2, \mathbb{K}}$ capitulate in $\mathbb{F}_{1}$, namely $[\mathcal{P}]$ and its square. Also the extension $\mathbb{F}_{1} / \mathbb{K}$ is of type (B), indeed, let $\mathbb{K}^{\prime}=\mathrm{k}\left(\sqrt{-q \varepsilon_{0} \sqrt{l}}\right)$, then we have $\mathbb{K} \mathbb{K}^{\prime}=\mathbb{F}_{1}$, since $N_{\mathbb{K} / \mathrm{k}}(\mathcal{P})=p$ and $p$ is unramified in $\mathbb{K}^{\prime} / \mathrm{k}$, then to show that $\mathcal{P}$ is inert in $\mathbb{F}_{1} / \mathbb{K}$, it suffices to show that $p$ is inert in $\mathbb{K}^{\prime} / \mathrm{k}$ (translation theorem) and for this we compute the norm residue symbol $\left(\frac{p,-q \varepsilon_{0} \sqrt{l}}{p}\right)$. Since $p \in \mathbb{Q}$ is inert in $\mathrm{k} / \mathbb{Q}$ and $-q \varepsilon_{0} \sqrt{l} \in \mathrm{k}$, therefore using the Proposition 4 , we find

$$
\left(\frac{p,-q \varepsilon_{0} \sqrt{l}}{p}\right)=\left(\frac{p, N_{\mathrm{k} / \mathbb{Q}}\left(-q \varepsilon_{0} \sqrt{l}\right)}{p}\right)=\left(\frac{p, l q^{2}}{p}\right)=\left(\frac{l}{p}\right)=-1,
$$

so $p$ is inert in $\mathbb{K}^{\prime} / \mathrm{k}$, which gives that $\mathcal{P}$ is inert in $\mathbb{F}_{1} / \mathbb{K}$, then $\mathbb{F}_{1} / \mathbb{K}$ is of type (B). Similarly, we show that extension $\mathbb{F}_{2} / \mathbb{K}$ is of type (B), using Theorem 1, only two classes of $C_{2, \mathbb{K}}$ capitulate in $\mathbb{F}_{2}$, namely [Q] and its square.

Using the notation of Theorem 4 , we have that $\left\{\xi, \xi^{\sigma}, \xi^{\sigma^{2}}\right\}$ is a fundamental system 
of units of $\mathbb{F}_{3}$, since $N_{\mathbb{F}_{3} / \mathbb{K}}(\xi)=N_{\mathbb{F}_{3} / \mathbb{K}}\left(\xi^{\sigma^{2}}\right)= \pm \varepsilon_{0}$ and $N_{\mathbb{F}_{3} / \mathbb{K}}\left(\xi^{\sigma}\right)= \pm \varepsilon_{0}^{-1}$, then we have $N_{\mathbb{F}_{3} / \mathbb{K}}\left(E_{\mathbb{F}_{3}}\right)=E_{\mathbb{K}}$, using Theorem 2, we found that only two classes of $C_{2, \mathbb{K}}$ capitulate in $\mathbb{F}_{3}$, namely $[\mathcal{P} Q]$ and its square. Moreover, the extension $\mathbb{F}_{3} / \mathbb{K}$ is of type (A), indeed, let $\mathbb{L}=\mathrm{k}\left(\sqrt{\varepsilon_{0} \sqrt{l}}\right)$, then we have $\mathbb{K} \mathbb{L}=\mathbb{F}_{3}, N_{\mathbb{K} / \mathrm{k}}(\mathcal{P})=p$ and $p$ is unramified in $\mathbb{L} / \mathrm{k}$, so to show that $\mathcal{P}$ is inert in $\mathbb{F}_{3} / \mathbb{K}$, it suffices to show that $p$ is inert in $\mathbb{L} / \mathrm{k}$, for this, we compute the norm residue symbol $\left(\frac{p, \varepsilon_{0} \sqrt{l}}{p}\right)$. We have $p \in \mathbb{Q}$ is inert in $\mathrm{k} / \mathbb{Q}$ and $\varepsilon_{0} \sqrt{l} \in \mathrm{k}$, so by Proposition 4 , we have $\left(\frac{p, \varepsilon_{0} \sqrt{l}}{p}\right)=$ $\left(\frac{p, N_{\mathrm{k} / \mathbb{Q}}\left(\varepsilon_{0} \sqrt{l}\right)}{p}\right)=\left(\frac{p, l}{p}\right)=\left(\frac{l}{p}\right)=-1$, therefore $p$ is inert in $\mathbb{L} / \mathrm{k}$, what gives that $\mathcal{P}$ is inert in $\mathbb{F}_{3} / \mathbb{K}$, and similarly one shows that $\mathcal{Q}$ remains inert in $\mathbb{F}_{3} / \mathbb{K}$ and like $\mathcal{P Q}$ capitulates in $\mathbb{F}_{3} / \mathbb{K}$, then by applying the Artin reciprocity law, we find that $\mathbb{F}_{3} / \mathbb{K}$ is of type (A), therefore, using Theorem 1 , the group $\mathbb{G}_{2}$ is isomorphic to $Q_{m}$ with $m>3$.

Example 1 . Let $\mathbb{K}=\mathbb{Q}(\sqrt{-55(4+\sqrt{17}) \sqrt{17}}), \mathbb{F}_{1}=\mathbb{K}(\sqrt{5}), \mathbb{F}_{2}=\mathbb{K}(\sqrt{-11})$ and $\mathbb{F}_{3}=\mathbb{K}(\sqrt{-55})$. By Theorem 5 , in each extension $\mathbb{F}_{i}$ there are exactly two classes of $C_{2, \mathbb{K}}$ which capitulate and $\mathbb{G}_{2} \simeq Q_{m}$ with $m>3$.

Corollary 2. With the same notation of Theorem 5 , we have $\# \mathbb{G}_{2}=4 h_{2}\left(\mathbb{K}_{0}\right)$ where $\mathbb{K}_{0}=\mathbb{Q}(\sqrt{l}, \sqrt{-p q})$ and $h_{2}\left(\mathbb{K}_{0}\right)$ its 2-class number.

Proof. We have $\mathbb{K}_{2}^{(1)} / \mathbb{F}_{3}$ is an unramified extension and the extension $\mathbb{F}_{3} / \mathbb{K}$ is of type $(A)$, then, according to [8], $C_{2, \mathbb{F}_{3}}$ is cyclic, thus $\mathbb{F}_{3}$ and $\mathbb{K}_{2}^{(1)}$ has the same Hilbert 2-class field which is $\mathbb{K}_{2}^{(2)}$, therefore $\# \mathbb{G}_{2}=2 h_{2}\left(\mathbb{F}_{3}\right)$. Moreover $\mathbb{F}_{3} / \mathrm{k}$ is a biquadratic normal extension with Galois group isomorphic to $\mathbb{Z} / 2 \mathbb{Z} \times \mathbb{Z} / 2 \mathbb{Z}$ and quadratic subfields $\mathbb{K}, \mathbb{K}_{0}$ and $\mathbb{L}=\mathrm{k}\left(\sqrt{\varepsilon_{0} \sqrt{l}}\right)$, so, by using [9], page 247 , we find that

$$
h_{2}\left(\mathbb{F}_{3}\right)=\frac{1}{2} q\left(\mathbb{F}_{3} / \mathrm{k}\right) h_{2}(\mathbb{K}) h_{2}\left(\mathbb{K}_{0}\right) h_{2}(\mathbb{L}),
$$

where $q\left(\mathbb{F}_{3} / \mathrm{k}\right)$ is the unit index of $\mathbb{F}_{3} / \mathrm{k}$ and $h_{2}(\mathbb{F})$ is the 2-class number of a number field $\mathbb{F}$. We have $h_{2}(\mathbb{K})=4$, according to [13] we have $h_{2}(\mathbb{L})=1$, by Corollary 1 we have $\left\{\varepsilon_{0}\right\}$ is a fundamental system of units of $\mathbb{K}$, using the Proposition 2 we show that $\left\{\varepsilon_{0}\right\}$ is a fundamental system of units of $\mathbb{K}_{0}$, according to Lemma 1 , we have $\left\{\xi, \xi^{\sigma}, \xi^{\sigma^{2}}\right\}$ is a fundamental system of units of $\mathbb{L}$ and $\mathbb{F}_{3}$, which gives that $q\left(\mathbb{F}_{3} / \mathrm{k}\right)=1$, thus $h_{2}\left(\mathbb{F}_{3}\right)=2 h_{2}\left(\mathbb{K}_{0}\right)$, therefore $\# \mathbb{G}_{2}=4 h_{2}\left(\mathbb{K}_{0}\right)$.

Corollary 3. Let $\mathbb{K}=\mathrm{k}\left(\sqrt{-p q \varepsilon_{0} \sqrt{l}}\right)$ where $\varepsilon_{0}$ is the fundamental unit of $\mathrm{k}=$ $\mathbb{Q}(\sqrt{l})$ with $l$ a prime number congruent to 1 modulo $8, p$ and $q$ be two primes such that $p \equiv-q \equiv 1 \bmod 4$ and $\left(\frac{p}{l}\right)=\left(\frac{q}{l}\right)=\left(\frac{p}{q}\right)=-1$, then $\mathbb{G}_{2} \simeq Q_{4}$. 
Proof. According to the Theorem 5, $\mathbb{G}_{2}$ is quaternionic of order $2^{m}$ with $m>3$. Let $\mathbb{K}_{0}=\mathbb{Q}(\sqrt{l}, \sqrt{-p q})$, since $\left(\frac{p}{l}\right)=\left(\frac{q}{l}\right)=\left(\frac{p}{q}\right)=-1$, then, according to [10], we have $h_{2}\left(\mathbb{K}_{0}\right)=4$, so, using Corollary 2, we find that $\mathbb{G}_{2} \simeq Q_{4}$.

Example 2. Let $\mathbb{K}=\mathbb{Q}(\sqrt{-15(4+\sqrt{17}) \sqrt{17}}), \mathbb{F}_{1}=\mathbb{K}(\sqrt{5}), \mathbb{F}_{2}=\mathbb{K}(\sqrt{-3})$ and $\mathbb{F}_{3}=\mathbb{K}(\sqrt{-15})$. According to the Theorem 5 , in each extension $\mathbb{F}_{i}$ there exist exactly two classes of $C_{2, \mathbb{K}}$ which capitulate and the group $\mathbb{G}_{2}$ is quaternionic of order $2^{m}$ with $m>3$. Furthermore, since $\left(\frac{5}{3}\right)=-1$, then, according to the Corollary $3, \mathbb{G}_{2} \simeq$ $Q_{4}$.

\section{REFERENCES}

[1] A. Azizi, "Sur les unités de certains corps de nombres de degré 8 sur QQ., Ann. Sci. Math. Québec, vol. 29, no. 2, pp. 111-129, 2005.

[2] A. Azizi and M. Talbi, "Capitulation dans certaines extensions non ramifiées de corps quartiques cycliques,” Archivum Mathematicum (BRNO), vol. 44, no. 4, pp. 271-284, 2008.

[3] E. Brown and C. J. Parry, "The 2-class group of certain biquadratic number fields ii," Pacific Journal of Mathematics, vol. 78, no. 1, pp. 11-26, 1978.

[4] M. N. Gras, "Table numérique du nombre de classes et des unités des extensions cycliques réelles de degré 4 de QQ," Publ. Math. Fac. Sciences de Besançon, Théorie des Nombres, vol. 2, pp. 1-79, 1977-78.

[5] H. Hasse, "Neue begründung und verallgemeinerung der theorie des normenrestsymbols," J. Reine Angew. Math., vol. 162, pp. 134-144, 1930.

[6] F. P. Heider and B. Schmithals, "Zur kapitulation der idealklassen in unverzweigten primzyklischen erweiterungen," J. Reine Angew. Math., vol. 366, pp. 1-25, 1982.

[7] M. Ishida, The genus fields of algebraic number fields, ser. Lecture notes in mathematics. London: Springer-Verlag, 1976, vol. 555.

[8] H. Kisilevsky, "Number fields with class number congruent to 4 modulo 8 and hilbert's theorem 94," J. Number Theory, vol. 8, no. 3, pp. 271-279, 1976.

[9] F. Lemmermeyer, "Kuroda's class number formula," Acta Arithmetica, vol. 66, no. 3, pp. 245-260, 1994.

[10] T. M. McCall, C. J. Parry, and R. R. Ranalli, "Imaginary bicyclic biquadratic fields with cyclic 2-class group," Journal of Number Theory, vol. 53, no. 1, pp. 88-99, 1995.

[11] K. Miyake, "Algebraic investigations of Hilbert's theorem 94, the principal ideal theorem and capitulation problem," Expos. Math., vol. 7, no. 4, pp. 289-346, 1989.

[12] H. Suzuki, “A generalisation of Hilbert's theorem 94," Nagoya Math. J., vol. 121, pp. 161-169, 1991.

[13] L. C. Washington, Introduction to cyclotomic fields (Book 83), 2nd ed., ser. Graduate texts in mathematics. New York: Springer-Verlag, 1997.

Authors' addresses

Abdelmalek Azizi

Mohamed First University, Department of Mathematics and Computer Sciences, Faculty of Sciences, Oujda, Morocco

E-mail address: abdelmalekazizi@yahoo.fr 
Mohammed Talbi

Regional Center of Education and Training, Oujda, Morocco

E-mail address: talbimmeyahoo.fr 